\title{
Cucullanus pargi sp. n. (Nematoda: Cucullanidae) from the grey snapper Lutjanus griseus off the southern coast of Quintana Roo, Mexico
}

\author{
David González-Solís ${ }^{1}$, Vielka M. Tuz-Paredes ${ }^{2}$ and Miguel A. Quintal-Loria ${ }^{2}$ \\ ${ }^{1}$ Laboratorio de Necton, El Colegio de la Frontera Sur (ECOSUR), Unidad Chetumal, Av. Centenario Km. 5.5, C.P. 77900, \\ A.P. 424, Chetumal, Quintana Roo, Mexico; \\ ${ }^{2}$ Instituto Tecnológico de Chetumal, Av. Insurgentes No. 330, C.P. 77013, A.P. 267, Chetumal, Quintana Roo, Mexico
}

Key words: Cucullanus pargi, parasitic nematode, Lutjanus griseus, Quintana Roo, Mexico

\begin{abstract}
A new nematode species, Cucullanus pargi sp. n., is described from the intestine and pyloric caeca of the grey snapper, Lutjanus griseus (Linnaeus), off the southern Quintana Roo coast, Mexico. This species shows similar morphological features as cucullanids occurring in marine and brackish-water fishes; however, it differs from all other species in the length of spicules, arrangement and number of caudal papillae, position of the excretory pore and deirids. Cucullanus pargi is the third species of this genus described from fishes in Mexico and the second one from Mexican marine fishes.
\end{abstract}

The genus Cucullanus Müller, 1777 is represented by an assemblage of species with a rather uniform morphology and many similarities (Moravec et al. 1993). The identification of species belonging to this genus is based particularly on the length and shape of the spicules, position of the excretory pore and deirids, and in the number and arrangement of caudal papillae. Petter (1974) pointed out that members of the genus Cucullanus are parasites of teleosts, amphibians and occasionally occur in turtles. The family Cucullanidae Cobbold, 1864 includes intestinal nematodes characterized by a highly developed buccal cavity formed by the oesophagus (oesophastome) (Berland 1970), and males with or without a precloacal sucker and harbouring 10-15 pairs of caudal papillae (Maggenti 1971). Most of about 100 species of Cucullanus have been described from fishes of different orders (Hasegawa et al. 1991) of which at least 70 were collected from marine or brackish-water fishes.

Studies on the nematode fauna of marine fishes are scarce in Mexico and all over the world. During recent helminthological examinations of specimens of the grey snapper Lutjanus griseus (Linnaeus) off the southern coast of Quintana Roo, nematodes belonging to the genus $\mathrm{Cu}$ cullanus were found. They differ from all related congeneric species, proving to represent a new species.

\section{MATERIALS AND METHODS}

A total of 360 specimens of the grey snapper were parasitologically examined. Fishes were bought from fishermen and sometimes they were caught by angling and by using throwing nets in 12 localities distributed along the Chetumal Bay and Caribbean Sea coast (Table 1). The collected nematodes were isolated and washed in physiological saline and fixed in hot $4 \%$ formaldehyde solution. They were cleared in glycerine for examination. Drawings were done with the aid of a drawing attachment. All measurements are given in millimetres.

\section{RESULTS}

Cucullanus pargi sp. n.

Fig. 1

Description. Medium-sized, yellowish nematodes. $\mathrm{Cu}-$ ticle thick and finely transversely striated. Cephalic end rounded, slightly dorsally bent. Buccal opening slit-like, dorsoventrally elongated. Narrow cuticular rim armed at its inner base with a row of numerous teeth and two pairs of papillae and one pair of amphids surrounding mouth. Oesophagus muscular, undivided, being expanded at its anterior and posterior parts forming pseudobuccal capsule (oesophastome) at its anterior end. Nerve ring encircling oesophagus between its first and second thirds. Deirids spine-like, near distal end of oesophagus or somewhat posterior to it. Excretory pore weakly visible, located posterior to oesophagus (Fig. 1A, B). Tail of both sexes conical, pointed (Fig. 1C, F, J).

Male (based on 6 specimens; measurements of holotype in parentheses): Body length 7.57-10.56 (9.53), maximum width $0.22-0.30(0.29)$. Oesophagus length $0.77-0.91$ (0.82), representing 9-10\% (9\%) of whole body length. Size of oesophastome, 0.22-0.27 (0.23) long and $0.15-0.21(0.19)$ wide. Width of posterior end of oesophagus $0.11-0.16(0.13)$. Nerve ring, deirids and excretory pore $0.28-0.34(0.28), 0.70-0.85(0.72)$ and $0.81-1.06$ (0.92), respectively, from anterior end of body. Precloacal sucker present. Distance of middle part of precloacal sucker to tip of tail $0.73-0.85$ (0.86). Cloacal lips somewhat elevated. Ventral muscular bands present. 

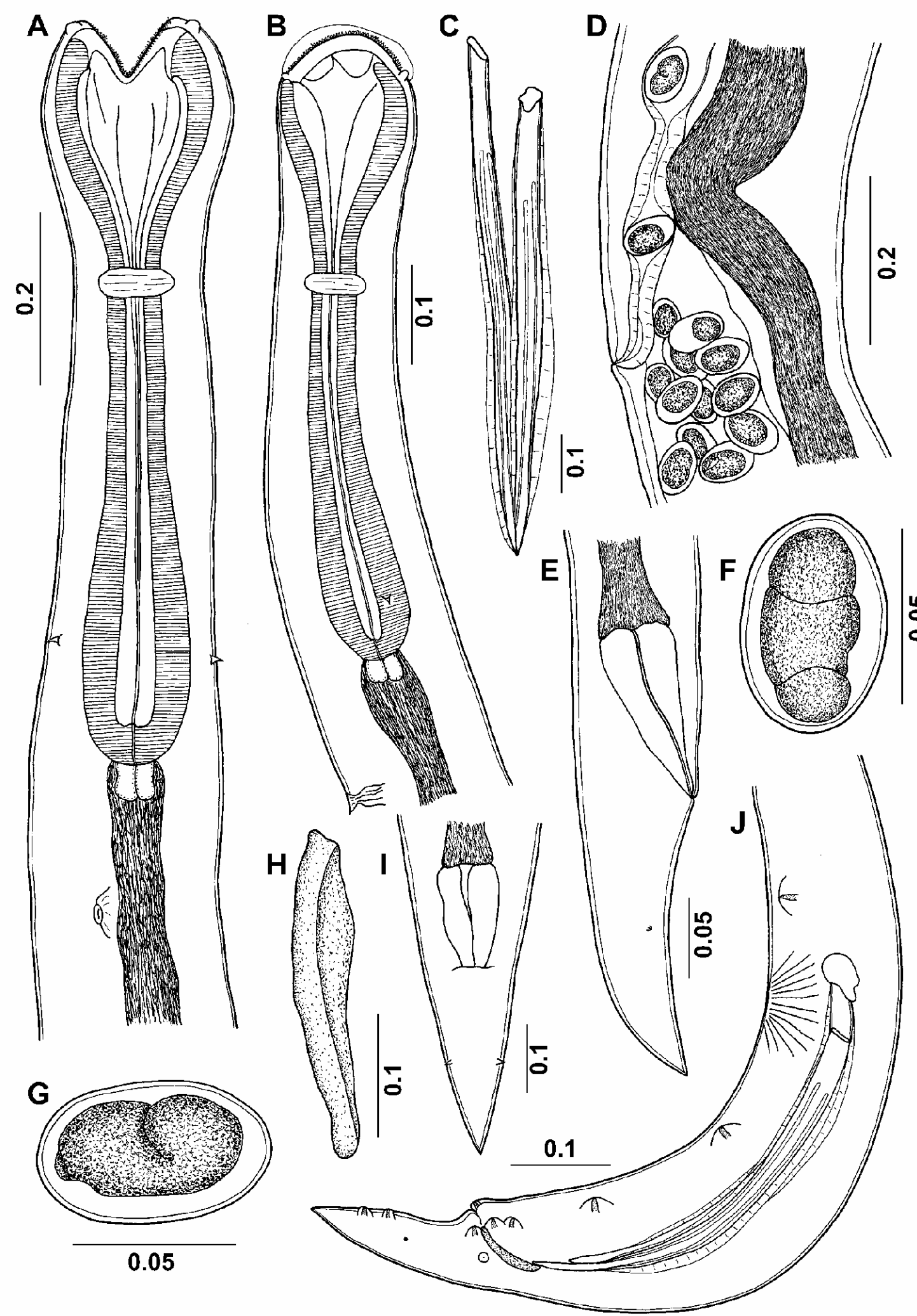

Fig. 1. Cucullanus pargi sp. n. A - anterior end of female, ventral view; $\mathbf{B}$ - anterior end of female, lateral view; $\mathbf{C}$ - spicules; $\mathbf{D}$ region of vulva; $\mathbf{E}$ - posterior end of female, lateral view; F, $\mathbf{G}$ - eggs; $\mathbf{H}$ - gubernaculum, lateral view; $\mathbf{I}$ - posterior end of female, ventral view; $\mathbf{J}$ - posterior end of male, lateral view. Scale bars in $\mathrm{mm}$. 
Table 1. Infection parameters of Cucullanus pargi sp. n. in fishes collected from different localities.

\begin{tabular}{lccccc}
\hline Localities & $\begin{array}{c}\text { Fish examined/ } \\
\text { infected }\end{array}$ & $\begin{array}{c}\text { Prevalence } \\
(\%)\end{array}$ & No. parasites & $\begin{array}{c}\text { Mean } \\
\text { abundance }\end{array}$ & $\begin{array}{c}\text { Mean } \\
\text { intensity }\end{array}$ \\
\hline Dos Hermanos & $48 / 15$ & 31 & 41 & 0.85 & 2.73 \\
La Aguada & $26 / 11$ & 42 & 28 & 1.07 & 2.55 \\
Río Krik & $22 / 7$ & 32 & 10 & 0.45 & 1.43 \\
Punta Calentura & $16 / 9$ & 56 & 16 & 1.00 & 1.78 \\
Tamalcab & $21 / 11$ & 52 & 33 & 1.57 & 3.00 \\
UQROO & $20 / 8$ & 40 & 19 & 0.95 & 2.38 \\
Punta Has & $25 / 13$ & 52 & 23 & 0.92 & 1.77 \\
Bacalar Chico & $49 / 12$ & 25 & 26 & 0.53 & 2.16 \\
Xcalak & $83 / 6$ & 7 & 8 & 0.09 & 1.33 \\
Tampalam & $15 / 3$ & 20 & 2 & 0.13 & 1.00 \\
Uvero & $18 / 2$ & 11 & 2 & 0.11 & 1.00 \\
Punta Gorila & $17 / 1$ & 6 & 1 & 0.06 & 1.00 \\
\hline
\end{tabular}

Three pairs of precloacal papillae, pair 1 anterior to precloacal sucker, pairs 2 and 3 posterior to sucker. Four pairs of adcloacal papillae close together; pairs 5, 6 and 7 subventral and pair 4 lateral. Latter located at level or slightly posterior to pair 7 of subventrals. Four pairs of postcloacal papillae (including phasmids), of which one subdorsal (pair 8), one lateral (phasmids) and two subventrals (pairs 9 and 10). Subdorsal papilla between subventral pairs or at level of the last pair. Unpaired papilla present on anterior cloacal lip (Fig. 1J). Spicules almost equal in size, right spicule 0.59-0.82 (0.78), left spicule 0.65-0.86 (0.81) long, well sclerotized. Spicules with narrow cuticular wing-like margin along almost its whole length; proximal end somewhat expanded, distal end pointed. Each spicule containing 2 inner supporting structures beginning at its mid-length (Fig. 1E). Gubernaculum 0.08-0.11 (0.09) long, well sclerotized (Fig. 1G). Tail conical, 0.18-0.25 (0.21) long, with pointed tip.

Female (based on 7 gravid specimens; measurements of allotype in parentheses): Body length 8.43-12.50 (11.87), maximum width $0.19-0.34$ (0.34). Oesophagus length $0.80-0.95(0.95)$, representing $8-10 \%(8 \%)$ of whole body length. Size of oesophastome, 0.22-0.27 (0.27) long and 0.16-0.21 (0.20) wide. Width of posterior end of oesophagus $0.09-0.14(0.13)$. Nerve ring, deirids and excretory pore $0.32-0.37(0.34), 0.70-0.90(0.90)$ and $0.82-0.93$ (0.93), respectively, from anterior end of body. Vulva postequatorial, 5.32-7.40 (7.40) from anterior end of body, with non-elevated lips. Vagina muscular, directed anteriorly from vulva (Fig. 1D). Uterus not extending anteriorly to base of oesophastome, occupying almost whole body cavity. Content of eggs in anterior and posterior parts of uterus not or slightly developed. Those at middle part of uterus and near vagina with $4-5$ blastomeres, 0.054-0.080 (0.076-0.080) $\times$ 0.038-0.060 $(0.056-0.060)$ in size (Fig. $1 \mathrm{H}, \mathrm{I})$. Ovary not extending posteriorly to anus. Tail conical, $0.28(0.28)$ long, with pointed end. Pair of lateral papillae (outlets of phasmids) present at mid-length of tail.

One juvenile specimen. Body length 5.44, maximum width 0.28 . Oesophagus length 0.75 , representing $14 \%$ of whole body length. Size of oesophastome 0.25 long and 0.17 wide. Width of posterior end of oesophagus 0.12 .
Nerve ring, deirids and excretory pore $0.30,0.74$ and 0.82 , respectively, from anterior end of body. Vulva postequatorial, 3.30 from anterior end of body, with non-elevated lips. Tail conical, 0.23 long, with pointed end.

$\mathrm{T}$ y $\mathrm{p}$ e h o s t: Grey snapper, Lutjanus griseus (Linnaeus, 1758) (Lutjanidae, Perciformes).

$\mathrm{S}$ ite of infection: Intestine and pyloric caeca.

$\mathrm{T}$ y p e 1 o c a 1 i t y : Xcalak, Quintana Roo, Mexico (18¹9'32”N, 8749'23”W) (date of collection: July 2004).

O t h e r 1 o c a 1 i t i e s : Dos Hermanos $\left(18^{\circ} 35^{\prime} 02^{\prime \prime} N\right.$,

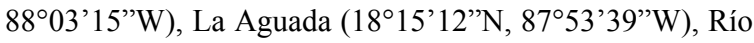
Krik (18 $\left.51^{\prime} 32^{\prime \prime N}, \quad 88^{\circ} 02^{\prime} 50^{\prime \prime} \mathrm{W}\right), \quad$ Punta Calentura $\left(18^{\circ} 28^{\prime} 45^{\prime \prime} \mathrm{N}, \quad 88^{\circ} 04^{\prime} 32^{\prime \prime} \mathrm{W}\right), \quad$ Tamalcab $\left(18^{\circ} 38^{\prime} 21^{\prime \prime} \mathrm{N}\right.$,

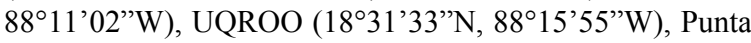
Has $\quad\left(18^{\circ} 23^{\prime} 37^{\prime \prime} \mathrm{N}, \quad 87^{\circ} 566^{\prime} 32^{\prime \prime} \mathrm{W}\right)$, Bacalar Chico $\left(18^{\circ} 11^{\prime} 41^{\prime \prime} \mathrm{N}, \quad 87^{\circ} 51^{\prime} 15^{\prime \prime} \mathrm{W}\right), \quad$ Tampalam $\left(1^{\circ} 09^{\prime} 20^{\prime \prime} \mathrm{N}\right.$,

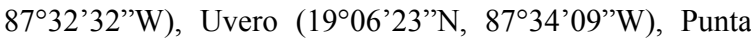

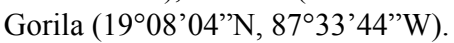

Infection parameters: see Table 1.

Deposition of specimens: Holotype and allotype in the Colección Nacional de Helmintos, UNAM, México (CNHE 5838). Paratypes in the Institute of Parasitology, ASCR, České Budějovice (No. N-875) and Colección de Parásitos de ECOSUR (ECOCH 053).

E t y m o log y: The specific name (in genitive) of this nematode relates to the common name of fish host "pargo" in the study region.

\section{DISCUSSION}

According to Petter (1974), a mouth perpendicular to the body axis, absence of intestinal caecum and sclerotized plaques in the pseudobuccal capsule are the most important features to allocate a nematode within the genus $\mathrm{Cu}$ cullanus. Since the nematodes of the present material possess all of these morphological traits, they have been allocated within this genus.

Most cucullanid species have rather uniform morphology and many have been inadequately described, therefore, a comparison among species is very complicated (Moravec et al. 1993). Apparently, cucullanids show narrow host specificity because the great majority of species have been found in phylogenetically related fishes. Taking 
this into account, the new species was directly compared with those species so far reported from lutjanid fishes around the world. At present only five species have been described from fishes of the family Lutjanidae: Dichelyne (Neocucullanellus) apharei Yamaguti, 1941 from Aphareus furcatus in Japan (Yamaguti 1941); Cucullanus sp. from Lutjanus sp. in Pakistan (Rasheed 1968); Cucullanus lutjani Schmidt et Kuntz, 1969 from Lutjanus gibbus and Gymnocranium griseus in the Phillipines (Schmidt and Kuntz 1969); Cucullanus rivulatus Soota et Dey Sarkar, 1980 from L. rivulatus in India (Soota and Dey Sarkar 1980); and Cucullanus bourdini Petter et Le Bel, 1992 from Pristipomoides filamentosus and Aprion virescens in New Caledonia and L. gibbus in the French Polynesia (Petter and Le Bel 1992, Morand and Rigby 1998). Of these species, $D$. (N.) apharei belongs to a different genus within the Cucullanidae. Cucullanus lutjani was later transferred to Dichelyne by Petter (1974), although considering its original description, Moravec et al. (1999) stated that it should be retained in the genus Cucullanus where it was originally listed. In this species, as in $C$. rivulatus, the precloacal sucker is absent, whereas $\mathrm{Cucul}$ lanus sp. was described from a single female (see Rasheed 1968). The new species shows similar patterns in the distribution of caudal papillae and position of deirids as $C$. bourdini. However, C. pargi differs in having larger body (males 10.6-14.0 vs. 7.57-10.56; females 18.5-21.7 vs. $8.43-12.50)$ and spicule (0.740-1.00 vs. $0.59-0.86)$ measurements and a more anterior location of pairs 4 and 8 of caudal papillae than $C$. bourdini. Many morphological features as the structure of the spicules, length of the gubernaculum, location of the excretory pore and allegedly absence of an unpaired papilla on the anterior cloacal lip were overlooked in the original description of the latter (see Petter and Le Bel 1992). However, the examination of paratype specimens allowed the determination of these morphological characters in specimens collected in New Caledonia, which are similar to those found during this survey.

Concerning the Cucullanus species from Perciformes, Petter (1974) reports 19 species, of which only 11 have a precloacal sucker. Additionally, 4 species with a precloacal sucker (C. carbonelli Campos, Carbonell et Rodrigues-Babio, 1993; C. carioca Vicente et Fernandes, 1973; C. cassinensis Pereira et da Costa, 1996; C. trachinoti Petter et Sey, 1997) have been described since Petter's work to date within this fish order. Out of these 15 species, C. lopholatilus (MacCallum, 1921) and C.cassinensis are considered as species inquirendae due to their poor descriptions (Petter 1974, Lanfranchi et al. 2004), whereas C. parvus Törnquist, 1931 apparently shows features related with the subgenus Cucullanellus as the presence of an intestinal caecum (Campos et al. 1993). Cucullanus micropapillatus Törnquist, 1931, C. carbonelli and C. amadai Yamaguti, 1940 show identical arrangement of caudal papillae but smaller body and spicule measurements, a not protuberant anus and a smaller gubernaculum.
Those species described from carangid fishes, C. pulcherrimus Barreto, 1918 and C. bulbosa (Lane, 1916), have only three pairs of adcloacal papillae and spicules are slightly smaller. The present specimens also show similar morphological features to those of $C$. trachinoti described from Trachinotus blochi from Kuwait by Petter and Sey (1997) and T. carolinus from Mexico by González-Solís et al. (2002). However, they differ in having somewhat smaller spicules, in the presence of papilla-like structures adjacent to the posterior subventral adcloacal papillae, arrangement of caudal papillae, and absence of unpaired cloacal papilla in $C$. trachinoti. All remaining species differ from the new species as follows: C. himezi Yamaguti, 1941 has larger body and tail measurements and different arrangement of caudal papillae; $C$. carioca has smaller body measurements; in C. sigani Yamaguti, 1954, the spicules and gubernaculum are longer; C. chrysophrydis Gendre, 1927 has spicules shorter than $0.5 \mathrm{~mm}$ and $C$. longicollis (Stossich, 1899) and C. mugili Belous, 1965 show smaller body sizes.

Since various species have been recorded from fishes belonging to different fish orders (Pleuronectiformes, Anguilliformes) (see Vicente and Santos 1973, Morand and Rigby 1998, Moravec et al. 2005), the new species was compared with Cucullanus species recovered from non-related hosts but with a precloacal sucker, similar arrangement of caudal papillae, and body and spicule measurements similar to those of the new species. Cucullanus heterochrous Rudolphi, 1802 has been mainly reported in Europe from marine fishes of the families Pleuronectidae and Soleidae (Moravec 1994) and differs by having slightly larger spicules and last two pairs of precloacal papillae close to each other. In $C$. hians Dujardin, 1845 , pair 4 is situated between pairs 6 and 7; pair 8 is in a lateral position instead of subdorsal and the unpaired adcloacal papilla is absent (see Timi and Lanfranchi 2006), whereas C. bonaerensis Lanfranchi, Timi et Sardella, 2004 has the excretory pore and deirids located in the second half of the oesophagus and the gubernaculum is smaller as well. Recently, C. oceaniensis Moravec, Sasal, Würtz et Taraschewski, 2005 was described from Anguilla spp. in Oceania (Moravec et al. 2005); it differs from the new species in the length of spicules and most posterior location of deirids.

This new species also differs from all other species of Cucullanus with a precloacal sucker, mainly in the distribution and number of caudal papillae, length of spicules, and in the position of the excretory pore. Therefore, we consider our specimens to represent a new species. So far, only two species of the genus Cucullanus have been described from freshwater (Caspeta-Mandujano et al. 2000) and marine (González-Solís et al. 2002) fishes in Mexico, thus $C$. pargi represents the second species recovered from marine fishes in Mexican waters.

Acknowledgements. The authors thank Roberto Herrera Pavón, Hannia M. Del Angel Coral and Francela Gallegos Torres for collecting and examining fishes. This survey was supported by Fondo Sectorial SAGARPA-CONACYT, project no. 48. 


\section{REFERENCES}

BERLAND B. 1970: On the morphology of the head in four species of Cucullanidae. Sarsia 43: 15-64.

CAmpos A., CARBonell M.D., RodrigueZ-BABIo C. 1993: Cucullanus carbonelli $\mathrm{n}$. sp. (Nematoda, Cucullanidae) parasite du poisson labridé Symphodus tinca. Vie Milieu 43: 225-229.

Caspeta-Mandujano J.M., Moravec F., Aguilar-Aguilar R. 2000: Cucullanus mexicanus sp. n. (Nematoda: Cucullanidae) from the intestine of the freshwater catfish Rhamdia guatemalensis (Pimelodidae) in Mexico. Helminthologia 37: 215 217.

GonZÁlez-Solís D., MoRAVEC F., VidAl-Martínez V.M., ZÁRATE-PÉREZ S.E. 2002: Parasitic nematodes of the Florida pompano, Trachinotus carolinus, from the Peninsula of Yucatán, Mexico. Helminthologia 39: 35-40.

HASEGAWA H., WiLliaMS E.H. JR., BUNKLEY-WILLIAMS L. 1991 Nematode parasites from marine fishes of Okinawa, Japan. J. Helminthol. Soc. Wash. 58: 186-197.

LANFRANCHI A.L., Timi J.T., SARDELla N.H. 2004: Cucullanus bonaerensis $\mathrm{n}$. $\mathrm{sp}$. (Nematoda: Cucullanidae) parasitising Urophycis brasiliensis (Pisces: Phycidae) from Argentinean waters. J. Parasitol. 90: 808-812.

MAGGENTI A.R. 1971: Review of the family Cucullanidae Cobbold, 1864 and the genus Bulbodacnitis Lane, 1916 with a description of Bulbodacnitis ampullostoma sp. n. (Nematoda: Cucullanidae) from Salmo gairdnerii Richardson. Proc. Helminthol. Soc. Wash. 38: 80-85.

MORAND S., RigBY M.C. 1998: Cucullanin nematodes from coral reef fishes of French Polynesia, with a description of Cucullanus faliexae n. sp. (Nematoda: Chitwoodchabaudiidae). J. Parasitol. 84: 1213-1217.

MORAVEC F. 1994: Parasitic Nematodes of Freshwater Fishes of Europe. Academia, Prague, $473 \mathrm{pp}$.

Moravec F., KoHn A., Fernandes B.M.M. 1993: Nematode parasites of fishes of the Paraná River. Brazil. Part 2. Seura-

Received 23 November 2006 toidea, Ascaridoidea, Habronematoidea and Acuarioidea. Folia Parasitol. 40: 115-134.

Moravec F., SASAl P., WÜRTZ J., TARASCHEWSKi H. 2005: Cucullanus oceaniensis sp. n. (Nematoda: Cucullanidae) from Pacific eels (Anguilla spp.). Folia Parasitol. 52: 343-348.

MoRAVEC F., WOLTER J., KÖRTING W. 1999: Some nematodes and acanthocephalans from exotic ornamental freshwater fishes imported into Germany. Folia Parasitol. 46: 296-310.

PetTer A.J. 1974: Essai de classification de la famille des Cucullanidae. Bull. Mus. Natl. Hist. Nat. Paris, series 3, Zoologie, 177: 1469-1491.

PetTer A.J., Le Bel J. 1992: Two new species in the genus Cucullanus (Nematoda: Cucullanidae) from the Australian region. Mem. Inst. Oswaldo Cruz 87: 201-206.

PetTER A.J., SEY O. 1997: Nematode parasites of marine fishes from Kuwait, with a description of Cucullanus trachinoti $\mathrm{n}$. $\mathrm{sp}$. from Trachinotus blochi. Zoosystema 19: 35-59.

RASHEED S. 1968: The nematodes of the genus Cucullanus Mueller, 1777, from the marine fish of Karachi coast. An. Esc. Nac. Cienc. Biol. Mex. 15: 23-59.

SCHMIDT G.D., KUNTZ R.E. 1969: Nematode parasites of Oceanica V. Four new species from fishes of Palawan, P.I., with a proposal for Oceanicucullanus gen. nov. Parasitology 59: 389-396.

SOOTA T.D., DEY SARKAR S.R. 1980: On three species of the nematode genus Cucullanus Mueller, 1777 and a note on Lappetascaris lutjani Rasheed, 1965, from Indian marine fishes. Rec. Zool. Surv. India 76: 1-6.

TIMI J.T., LANFRANCHI A.L. 2006: A new species of Cucullanus (Nematoda: Cucullanidae) parasitizing Conger orbignianus (Pisces: Congridae) from Argentinean waters. J. Parasitol. 92: 151154.

ViCENTE J.J., SANTOS E. 1973: Alguns helmintos de peixe do litoral norte fluminense. Mem. Inst. Oswaldo Cruz 71: 95-113.

YAMAGUTI S. 1941: Studies on the helminth fauna of Japan. Part 33. Nematodes of fishes, II. Jpn. J. Zool. 9: 343-396.

Accepted 26 March 2007 